\title{
MEETING PROGRAM
}

\section{Monday, June 2}

arrival during the afternoon, transfer to the different Hotels, Hotel check-in, meeting registration dinner (Hotels)

\section{Tuesday, June 3}

8.00: meeting registration

8.30: ouverture de la journée SFTA, accueil par P. Mura

8.30: SoHT Workshop opening, welcome address by C. Jurado and P. Kintz

8.30 - 10.30: TIAFT Young Scientists meeting

8.45 - 10.30: session Toxicologie hospitalière, $\mathbf{1}^{\text {ère }}$ partie (SFTA)

8.45 - 10.30: session Analytics in hair testing (SoHT)

10.30 - 11.00: coffee break sponsored by Siemens-Dade Behring

11.00 - 12.30: TIAFT Young Scientists meeting

11.00 - 12.30: session Toxicologie hospitalière, $2^{\text {ème }}$ partie (SFTA)

11.00 - 12.30: session Alcohol markers (SoHT)

12.30 - 13.45: lunch for participants and accompanying persons sponsored by Waters

13.45 - 16.15: session Toxicologie environnementale et professionnelle (SFTA)

13.45 - 15.30: session Interpretation and applications of hair analysis (SoHT)

15.30 - 16.30: coffee break

16.30 - 17.30: SoHT business meeting

17.30 - 19.30: welcome reception sponsored by Agilent Technologies

dinner (Hotels)

(salle Sapotille)

(room Lafitte)

(room Tamarin)

(salle Sapotille)

(room Lafitte)

(room Tamarin)

(salle Sapotille)

(room Lafitte)

(Hotel La Batelière)

(salle Sapotille)

(room Lafitte)

(beach Hotel la Batelière)

\section{Wednesday, June 4}

8.30 - 10.00: TIAFT Congress opening ceremony

Welcome address on behalf of SFTA

Welcome address on behalf of SoHT

Welcome address on behalf of TIAFT

Have fun in La Martinique, some short stories from: Marilyn Huestis, Hans Maurer, Marc LeBeau,

Dan Isenschmid, Annette Akgur, Manfred Moeller and Carmen Jurado

Special lecture 1 (20 min):Robert Wennig

Special lecture 2 (20 min): Jean Pierre Anger, Pascal Kintz

10.00 - 10.30: coffee break, sponsored by Immunalysis

10.30 - 12.30: session Postmortem toxicology

12.30 - 13.45: lunch for participants and accompanying persons sponsored by Abbott Diagnostics

13.45 - 15.45: session Analytical aspects, part 1

15.45 - 16.15: coffee break, sponsored by Immunalysis

16.15 - 17.30: session Occupational toxicology

18.00: fun on the beach with Zouk lessons, dinner (Hotels) sponsored by Siemens-Dade Behring

S1-VI

Article available at http://www.ata-journal.org or http://dx.doi.org/10.1051/ata:2008015 (rooms Lafitte and Sapotille)

Patrick Mura

Carmen Jurado

Pascal Kintz

"History of TIAFT"

"Is rum toxic?"

(Hotel la Batelière)

(beach Hotel la Batelière) 


\section{Thursday, June 5}

8.30 - 10.15: sponsored workshops 1 and 2

by Thermo Fisher Scientific (room Lafitte) and Roche Diagnostics (room Sapotille)

10.00 - 10.30: coffee break

10.30 - 12.00: sponsored workshops 3 and 4

by Waters (room Lafitte) and Inverness Medical Innovation

$10.00-12.00$ : local games and sport activities

$12.30-13.45$ : lunch for participants and accompanying persons

14.00 - 15.30: sponsored workshops 5 and 6

by Applied Biosystems (room Lafitte) and Randox Laboratories (room Sapotille)

16.00: trip to Habitation - Rhumerie Clément by bus

(departure from Hotel la Batelière) sponsored by Waters

Caribbean dinner at Habitation Réunion and return by bus to the Hotels, sponsored by Waters

(salle Sapotille)

\section{Friday, June 6}

8.00 - 10.15: session Clinical Toxicology and Pharmacology (joint session with IATDMCT) (rooms Lafitte and Sapotille) 8.00 - 17.30: exhibition with Agilent Technologies, Waters, Applied Biosystems, Thermo Fisher Scientific,

Roche Diagnostics, Medichem, Varian, Lipomed, Randox Laboratories, Chromsystems Instruments \& Chemicals,

LGC Standards, Siemens-Dade Behring

(rooms Tamarin and Corossol)

8.00 - 17.30: poster session

(auditorium)

10.15 - 10.45: coffee break, sponsored by Waters

10.45 - 12.45: session New trends in DUI detection

12.45 - 14.00: lunch for participants and accompanying persons

(Hotel la Batelière)

14.15 - 15.30: session Analytical aspects, part 2

15.30 - 16.30: session Free topics

16.30 - 17.00: coffee break, sponsored by Chromsystems

17.00 - 18.30: sponsored workshops 7 and 8

dinner (Hotels)

by Agilent (room Lafitte) and Siemens (room Sapotille)

\section{Saturday, June 7}

9.00 - 10.45: session Alternative specimens

(rooms Lafitte and Sapotille)

9.00 - 17.30: exhibition with Agilent Technologies, Waters, Applied Biosystems, Thermo Fisher Scientific,

Roche Diagnostics, Medichem, Varian, Lipomed, Randox Laboratories, Chromsystems Instruments \& Chemicals,

LGC Standards, Siemens-Dade Behring

(rooms Tamarin and Corossol)

9.00 - 17.30: poster session

(auditorium)

10.45 - 11.15: coffee break

11.15 - 12.15: session Drugs of abuse and seized material

12.30 - 13.45: lunch for participants and accompanying persons

(Hotel La Batelière)

14.00 - 16.00: TIAFT business meeting

16.00 - 16.30: coffee break

19.30: gala dinner SFTA/TIAFT with Zouk dance, sponsored by Thermo Fisher Scientific

(Hotel Bakoua, Trois Ilets, transportation by boat)

\section{Sunday, June 8}

lunch at your convenience

return, transfer to the airport 